\title{
Very Long Baseline Interferometry (VLBI)
}

\author{
R.T. Schilizzi, Dwingeloo
}

\author{
(Netherlands Foundation for Radio Astronomy)
}

Very Long Baseline Interferometry (VLBI) is an advanced technique in astronomy coupling radio telescopes in different nations together to form a single radio telescope of continental, or even Earth-sized dimensions. VLBI networks, with their effective diameters of up to $8000 \mathrm{~km}$, are the largest telescopes which have ever probed the depths of the Universe and can achieve sub-milliarcsecond angular resolution. This is 1000 times better than for groundbased optical telescopes and 100 times better than for the 2.4 m diameter Hubble Space Telescope. Details can be seen in cosmic radio sources one hundred thousand times smaller than is possible with the individual telescopes in the system. The data gathered provide information on galaxies, quasars, and stars on the smallest scales ever directly measured, information that is crucial to our understanding of the most energetic phenomena in Nature's laboratory.

VLBI is at the same time able to measure the distances between points on the Earth, 100 s to 1000 s of kilometres apart with accuracies of a few centimetres, and is one of the two main techniques in use by geophysicists and geodesists for studying continental drift and regional crustal movements.

\section{Principles and Practice}

The extremely weak signals (typically $10^{-18} \mathrm{~W}$ ) from a distant radio object are received at the individual elements (telescopes) of an interferometer system, amplified, translated in frequency to a low frequency band, and then sent to a special purpose correlator where they are multiplied together in pairs. The process of multiplication searches for the fraction of the total signal which is correlated between the different elements of the interferometer.

The further apart the elements of the interferometer, the smaller in size the radio object may be that will produce a measurable correlated signal. An image of the object is built up by measuring the correlated signal for many different interferometer pairs, followed by a mathematical (Fourier) transformation in a high speed digital computer (a so-called X-F correlator). A variant of this process involves Fourier transformation followed by cross-correlation (an F-X correlator). Seen from a radio source, an interferometer on Earth has an effective spacing and orientation determined by its projection perpendicular to the direction to the source. The projected spacing of the interferometer is sensitive to a particular scale of structure in the source, which is equivalent to saying it measures a particular spatial frequency in the Fourier transform of the intensity distribution of the source. With a sparsely filled aperture as we have in VLBI, it is essential to increase the number of spatial frequencies sampled by an interferometer in order to reconstruct reliably images of complex sources. The rotation of the Earth is used to this end, as is shown in Fig. 1.

Here the length and orientation of the baseline between two telescopes, as seen from the source, is shown as a vector from the origin which rotates and

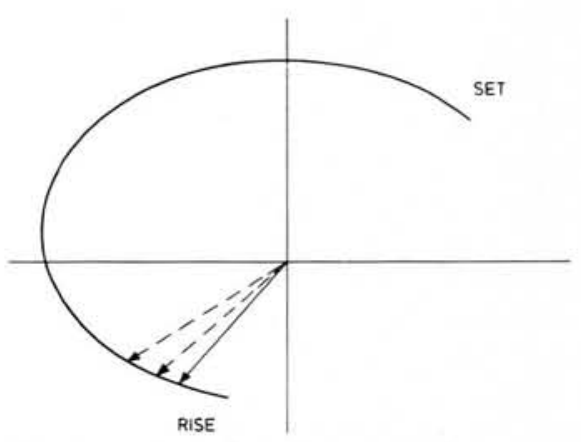

Fig. 1 - A track in the spatial frequency (U-V) plane of an interferometer. The curve traces out the locus of the changing projection, due to the Earth's rotation, of the baseline between two telescopes, as seen from a radio source.

changes length as the earth rotates. The curve begins when the source rises for the western antenna and ends when it sets for the eastern antenna. Each point on the curve represents a different interferometer, measured in sucession. The same curve would be produced if we actually had telescopes for all those interferometers and took one measurement

Fig. 2 - A schematic diagram of a two-element VLB interferometer.

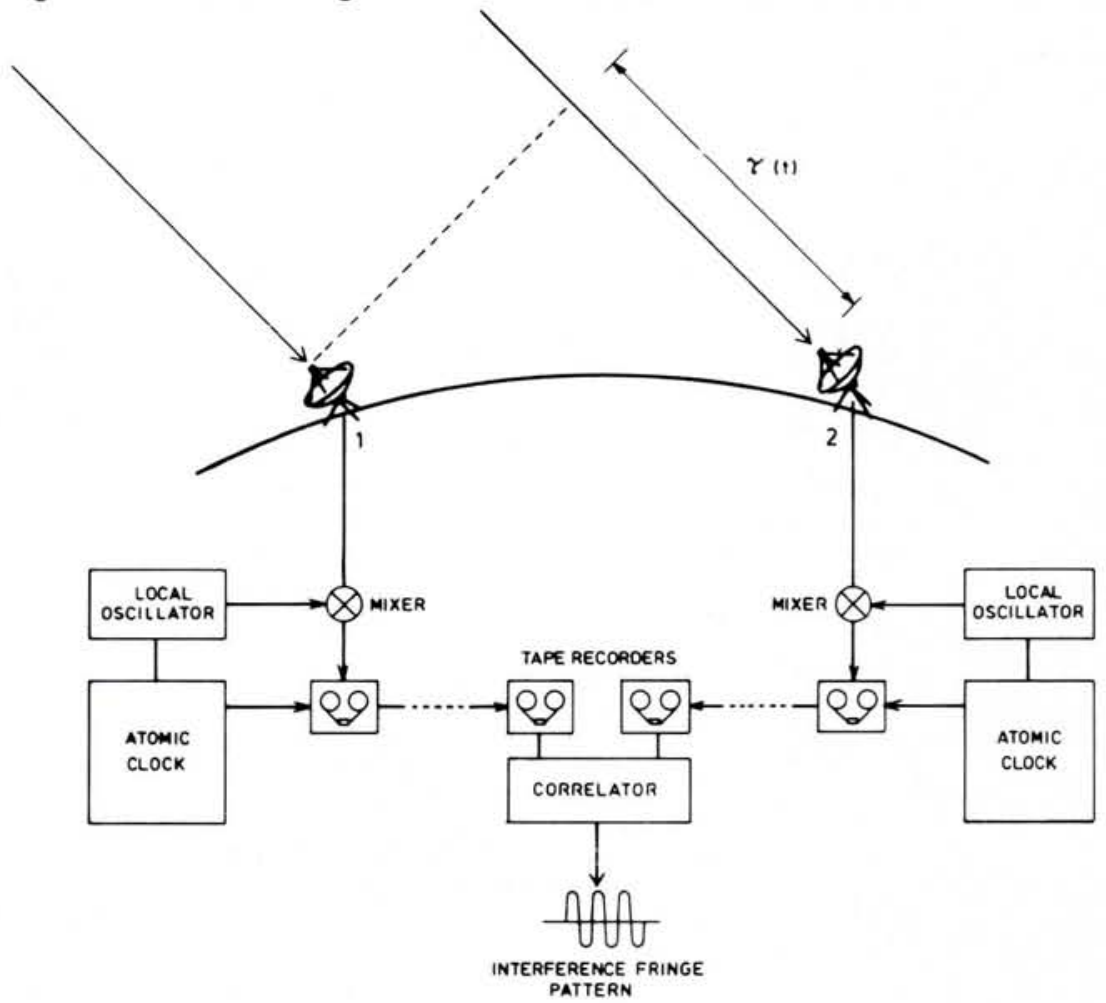


in time with the array. When there is a network of antennas, the diagram becomes far more filled. The better filled the spatial frequency, or U-V plane, the better image quality. The details which can be distinguished in this image are as fine as those which would have been observed with a single telescope having a diameter as large as the largest separation of telescopes. This technique is called "Earth-Rotation A perture Synthesis" and is the basis for VLBI and for "connected" interferometers with smaller dimensions.

Present day interferometers come in many sizes, with separations ranging from a few tens of metres up to the size of the Earth. For separations up to a few tens of kilometres, cables or waveguides can be used to send a common signal for frequency translation out to the individual telescopes of the interferometer and to conduct the signals received at the individual telescopes from the radio source back to the central correlator.

Beyond this, up to about $150 \mathrm{~km}$, radio links can be used, but at even greater separations, any form of real-time connection becomes prohibitively expensive for research institute budgets.

VLBI overcomes this problem by utilising two modern technological developments. The first is the use of atomic frequency standards, usually hydrogen maser oscillators, at each radio telescope to produce independent frequency translation signals that are similar enough to each other that they are equivalent to the required "common signal". The second is the use of professional video tape recorders at each telescope to record the incoming signal, after frequency translation, on magnetic tape. Bandwidths of up to $56 \mathrm{MHz}$ are recorded on current systems, increasing to perhaps $256 \mathrm{MHz}$ in the system now under development. The tapes are then delivered to the VLBI data processing facility and played back through the correlator in the same way as if there had been direct connections from the individual telescopes. The difference is that the playback occurs days to weeks after the observation instead of in real time.

A schematic diagram of a simple twoelement VLB interferometer is shown in Fig. 2. The greater the number of telescopes in the interferometer system the more nearly does it fully synthesize the large aperture and the higher is the quality of the image reconstruction. VLBI systems have progressed from simple two or three telescope configurations in the early 1970's to world-wide arrays of up to eighteen telescopes in the current era.

\section{\begin{tabular}{|l|l|} 
FU & BERLIN \\
\hline
\end{tabular}}

\section{Professorship in Experimental Physics}

The Department of Physics at the Freie Universität Berlin announces an opening for a

\section{PROFESSOR POSITION (C 3)}

in the field of experimental physics.

The applicant is expected to engage in research in solid state physics using nuclear methods. For this purpose the heavy-ion accelerator VICKSI of the Hahn-Meitner-Institute (HMI) is available. The applicant is furthermore expected to participate in the teaching duties of the Department.

To apply, please send curriculum vitae, list of publications, teaching records, and names of reference to the

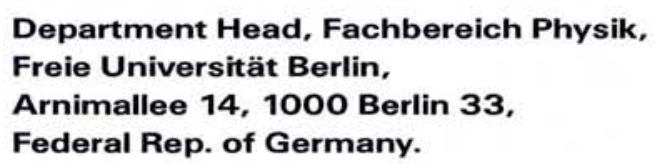

Special image processing techniques have been developed for VLBI which have had wide-ranging application in radio interferometry in general and are now being applied in optical telescopes for image sharpening. These techniques are successful to a large extent in removing errors in the data caused by tropospheric and ionospheric fluctuations above the individual telescopes as well as differences in the performance of the atomic frequency standards. tance-measuring devices for geodesy. $A$ VLB interferometer measures the diffe-
VLBI systems are also accurate dis- rence in arrival time of the signals at individual telescopes with precisions of better than one tenth of one nanosecond. This corresponds to measuring differences in the path length travelled by the signal from the radio source to the telescopes to better than $5 \mathrm{~cm}$. Since the precision in measuring the difference in arrival time is independent of the distance between the telescopes in the interferometer, a VLBI system can measure intercontinental distances to precisions of $5 \mathrm{~cm}$ or better. It has been used in this context to track continental drift and local tectonic motion.

\section{The FOM-Institute for Plasma Physics "Rijnhuizen", Nieuwegein, The Netherlands, invites highly qualified applicants for the position of Head of the Theory Division}

The scientific programme of the theory division is concentrated on the theory of hot, magnetized plasmas (Fusion Plasmas) and of free electron lasers. It involves studies that have both an intrinsic theoretical value and are relevant to the experimental programme of the Institute. The entire programme is part of the European Fusion Programme (Euratom).

The theory group consists of five senior physicists, two junior physicists and two Ph.D. candidates.

Applicants should have an extensive internationally recognized experience in theoretical plasma physics.

Applications including a curriculum vitae, a list of publications and the names and addresses of two references should be addressed to:

Prof. M.J. van der Wiel, Institute for Plasma Physics,

Postbus 1207, NL-3430 BE Nieuwegein, The Netherlands.

The salary will be according to the Dutch Civil Servants Code: BBRA Scale 16, with a maximum of HFL 9621.- per month. Further information may be obtained from the director of the Institute: Prof. M.J. van der Wiel, Tel.: (3402) 31224.

The Closing Date is 22 May, 1988. 


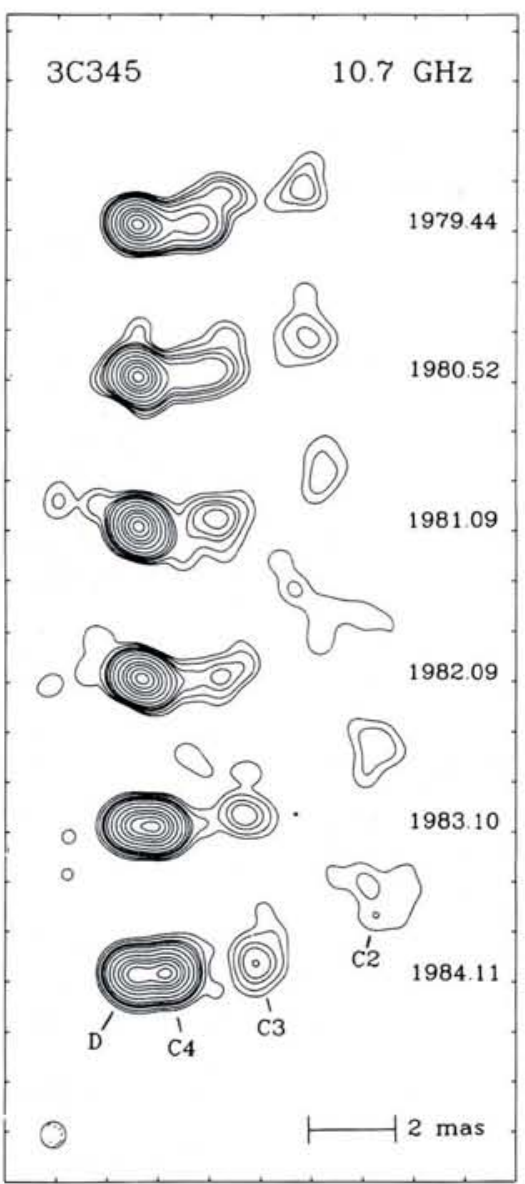

Fig. 3-Maps of $3 \mathrm{C} 345$ at $10.7 \mathrm{GHz}$ (Ref. 1). North is at the top; tick marks are at spacings of 1.2 milli-arcsec (mas).

\section{VLBI Networks in the 1980's}

One of the most challenging aspects of VLBI is the smooth execution of the same observing programme at widely separated radio telescopes. Both in the USA and in Europe there exist VLBI Networks with well-defined management structures to enable astronomers anywhere in the world to obtain VLBI data. Geodesists have also set up (largely) independent networks for their own specific purposes. At least $25-30 \%$ of all observing time on the member telescopes is given over to VLBI, and in a growing number of institutes, $100 \%$ of the time.

The European VLBI Network was established in 1980 with five full member institutes and four associate members (Fig. 5 and Table 1). In 1984, the member institutes associated themselves in the European Consortium for VLBI. The Consortium is governed by a Board of Directors consisting of the Institute Directors, which delegates the operation of the EVN to three committees, the Programme Committee which meets three times a year to select observing proposals, the Technical Working Group which coordinates instrumental developments in the EVN, and a network of "friends of VLBI" at each telescope to carry out the observing programmes. Scheduling of the EVN observing sessions and coordination of the activities of the friends of VLBI during the sessions is the responsibility of the Chairman of the Programme Committee. The Board of Directors recently appointed a Network Scientist to look into means for improving the scientific performance of the EVN, and a Project Manager to oversee efforts to develop new generation processing facilities. A similar, if not identical management structure is found in the US VLBI Network.

Astronomers submit proposals for observations at frequencies between $327 \mathrm{MHz}$ and $22 \mathrm{GHz}$, which are reviewed by programme committees, and time is assigned or not depending on the collective or collected opinion of the referees. In the last four or five years an increasing number of proposals have been so-called "global" proposals involving telescopes in both networks and elsewhere, up to a maximum, so far, of 18 antennas. These observations have to be carefully coordinated around the world, but that is now relatively routine.

\section{Highlights of VLBI Astronomy}

Some of the most interesting and exciting phenomena in the Universe are those associated with high energy $\left(10^{33}\right.$ $-10^{38} \mathrm{~J} / \mathrm{s}$ ) processes taking place in the centres of astronomical objects. High resolution VLBI measurements probing these central power houses are the only means at present to generate pictures of the emitting regions, and, as such, are extremely valuable in extending our knowledge of physical processes at very high energies.

Superluminal motion in quasars and active galactic nuclei - evidence for matter moving at almost the speed of light.

One of the earliest discoveries made with VLBI was evidence found in 1969 for apparent separations of components in compact radio sources at speeds greater than that of light. Confirmation of this astonishing result was finally established as VLBI imaging capabilities developed in the 1970s. An example can be seen in Fig. 3 where a sequence of observations of the quasar $3 \mathrm{C} 345$ over five years is shown. The apparent velocities derived for the various components of the structure range from 1.3 to 9.5 times the speed of light.

The phenomenon of superluminal motion has led to many imaginative theories attempting to explain it as an illusion. The most widely discussed model invokes special relativistic kinematics for components moving away from a stationary "core". Consider a source of radio radiation morning away from a core with a bulk relativistic velocity of $v$ $=\beta c$ towards the observer at angle $\theta$ to

Table 1: Member institutes of the European Consortium for VLBI

\begin{tabular}{|c|c|c|c|c|}
\hline Institute & $\begin{array}{l}\text { Membership } \\
\text { Status }\end{array}$ & $\begin{array}{l}\text { Telescope } \\
\text { Location }\end{array}$ & $\begin{array}{l}\text { Telescope } \\
\text { Diameter }\end{array}$ & Comment \\
\hline $\begin{array}{l}\text { Nuffield Radio Astronomy Lab., } \\
\text { Jodrell Bank, UK }\end{array}$ &. & Jodrell Bank & $\begin{array}{l}76 m \\
26 m\end{array}$ & \\
\hline $\begin{array}{l}\text { Paris Observatory, } \\
\text { Meudon, France }\end{array}$ & A & Nançay & $94 \mathrm{~m}$ & $\begin{array}{l}\text { (equivalent } \\
\text { diameter) }\end{array}$ \\
\hline $\begin{array}{l}\text { Netherlands Foundation } \\
\text { for Radio Astronomy, } \\
\text { Dwingeloo, NL }\end{array}$ & $\mathrm{F}$ & Westerbork & $93 \mathrm{~m}$ & (phased array) \\
\hline $\begin{array}{l}\text { Max-Planck-Institut } \\
\text { for Radio Astronomy, } \\
\text { Bonn, FRG }\end{array}$ & $\mathrm{F}$ & Effelsberg & $100 \mathrm{~m}$ & \\
\hline $\begin{array}{l}\text { Institute of Radio Astronomy, } \\
\text { Bologna, Italy }\end{array}$ & $\mathrm{F}$ & $\begin{array}{l}\text { Medicina } \\
\text { Noto } \\
\text { Sardinia }\end{array}$ & $\begin{array}{l}32 \mathrm{~m} \\
32 \mathrm{~m} \\
\geq 32 \mathrm{~m}\end{array}$ & $\begin{array}{l}\text { dedicated to VLBI } \\
\text { 1988, dedicated } \\
>1988 \text {, dedicated }\end{array}$ \\
\hline $\begin{array}{l}\text { Chalmers Technical Univ., } \\
\text { Goteborg, Sweden }\end{array}$ & $\mathrm{F}$ & Onsala & $\begin{array}{l}26 \mathrm{~m} \\
20 \mathrm{~m}\end{array}$ & \\
\hline $\begin{array}{l}\text { Technical University of Munich, } \\
\text { University of Bonn, FRG }\end{array}$ & A & Wettzell & $20 \mathrm{~m}$ & $\begin{array}{l}\text { dedicated to VLBI } \\
\text { geodesy }\end{array}$ \\
\hline $\begin{array}{l}\text { Nicolaus Copernicus University, } \\
\text { Torun, Poland }\end{array}$ & $y$, & Torun & $\begin{array}{l}15 \mathrm{~m} \\
32 \mathrm{~m}\end{array}$ & $\begin{array}{l}\text { 1990, dedicated } \\
\text { to VLBI }\end{array}$ \\
\hline $\begin{array}{l}\text { Space Research Institute, } \\
\text { Moscow, USSR }\end{array}$ & A & $\begin{array}{l}\text { Simeis } \\
\text { Suffa }\end{array}$ & $\begin{array}{l}22 \mathrm{~m} \\
70 \mathrm{~m}\end{array}$ & $\begin{array}{l}\text { 1990, dedicated } \\
\text { to VLBI }\end{array}$ \\
\hline $\begin{array}{l}F: \text { full member } \\
A: \text { associate member }\end{array}$ & & & & \\
\hline
\end{tabular}




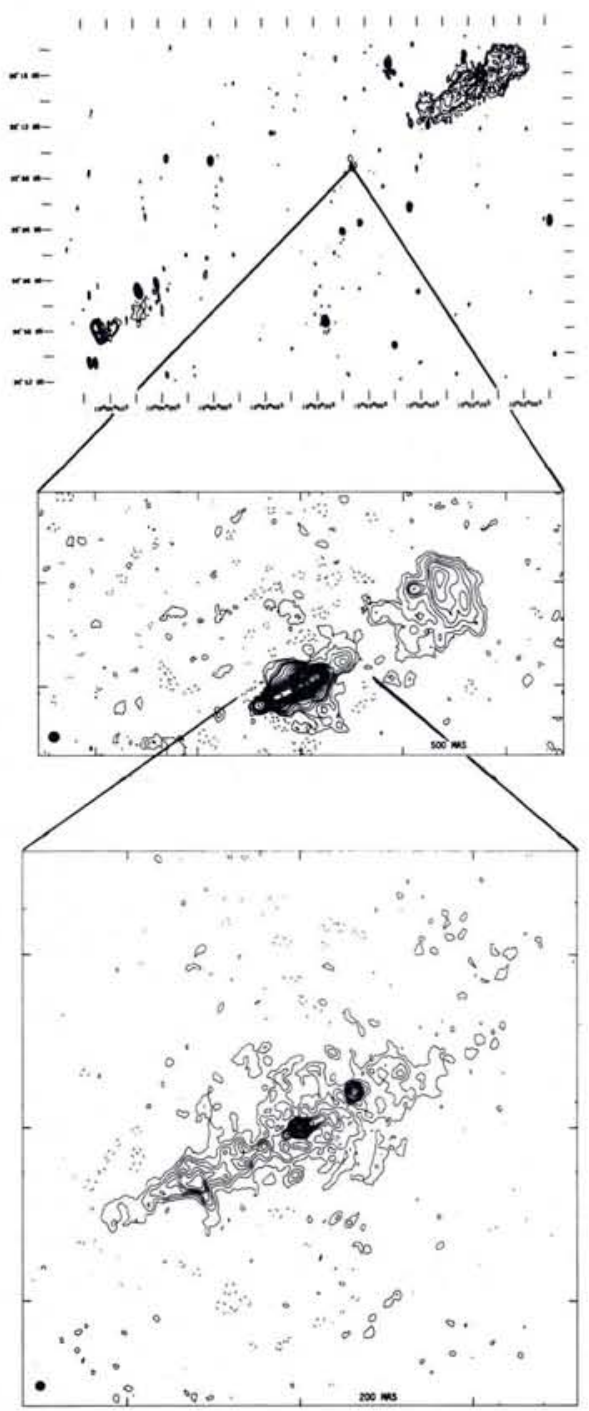

Fig. 4 - The radio structure of the radio galaxy $3 C 236$ on scales from 45 light years to 12 million light years (Ref. 2). 3 C236 is at a distance of 900 million light years. Top panel: Westerbork $21 \mathrm{~cm}$ map; overall width 3 arcmin. Middle panel: VLBI + MERLIN 18 $\mathrm{cm}$ map width 2500 mas. The linear extent of the nuclear structure is 7000 light years. Bottom panel: VLBI + MERLIN $18 \mathrm{~cm}$ map showing a two-sided jet close to the nucleus which is the bright feature in the middle; width 600 mas.

the line of sight. The observed transverse velocity of separation is:

$$
v_{\text {app }}=\beta_{\text {app }} c
$$

where $\beta_{\text {app }}=(\beta \sin \theta) /(1-\beta \cos \theta)$. The factor $\beta_{\text {app }}$ can exceed unity for smallish $\theta$ and $\beta \rightarrow 1$, so that $v_{\text {app }}>c$ and we have faster-than-light motion. This model can also explain rapid flux density variations, the one-sidedness of the emission, the apparent rotation of the radio structure as it moves away from the core and the fact that the observed $\mathrm{X}$-ray emission is much weaker than expected from the synchrotron selfCompton process (see the many papers in Superluminal Radio Sources (Cambridge Univ. Press) 1987). The obser- vable characteristics of relativistically moving sources of radiation depend strongly on the viewing angle of the observer with respect to the emission axis. Emitting regions approaching at relativistic velocity close to our line-ofsight tend to appear much more luminous through Doppler enhancement, and hence to be over-represented in radio source surveys. The appearance of the radio sky may in fact be largely dominated by these relativistic effects, so that many (if not most) of the strongest compact sources in the sky are likely to be superluminal. This expectation is borne out by recent observations.
Alignment of small scale and large scale jets in active galactic nuclei - evidence for strong collimation mechanisms in the centres of galaxies and quasars.

In powerful radio sources the compact nuclear components imaged by VLBI are found to have the same orientation in the sky as the large scale extended lobes (see Fig. 4). This demonstrates that the initial collimation producing this alignment takes place on scales of less than about one light-year in the nucleus and is probably the result of the shape of the accretion disk around a massive black hole in the centre whose mass is $10^{6}$ to $10^{9}$ times that of the Sun.

\section{CAMBRIDGE MONOGRAPHS ON MATHEMATICAL PHYSICS \\ Superstring Theory \\ MICHAEL B. GREEN, JOHN H. SCHWARZ and EDWARD WITTEN} '... likely to remain standard reference works for years to come; certainly there is
no serious rival at present.'
Nature

Volume 1: Introduction $\quad 469$ pp. $1988 \quad 052135752 \quad 7 \quad$ Pb $\$ 15.00$ net Volume 2: Loop Amplitudes, Anomalies and Phenomenology

$596 p p .1988 \quad 0 \quad 521357535 \quad$ Pb $\$ 17.50$ net

\section{Group Structure of Gauge Theories}

\section{LOCHLAINN O'RAIFEARTAIGH}

'Altogether, the book is a welcome addition to the literature in this field, by a real expert, and will repay the closest study. It is especially an excellent book for graduate students in physics.'

Science

$$
172 \text { pp. } 1988 \quad 0 \quad 521 \quad 34785 \quad 8 \quad \text { Pb } \$ 8.95 \text { net }
$$

\section{Functional Integrals and Collective Excitations}

\section{N. POPOV}

Describes the concepts and selected applications of one of the most important mathematical tools used in the theoretical investigation of collective excitations in

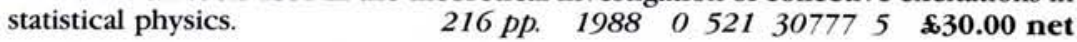

\section{Path Integral Methods in Quantum Field Theory}

\section{R. J. RIVERS}

A concise graduate-level introduction to analytic functional methods in quantum field theory. $\quad 339$ pp. $1987 \quad 0 \quad 521 \quad 259797 \quad \mathbf{8 4 2 . 5 0}$ net

\section{Gravitational Physics of Stellar and Galactic Systems}

WILLIAM C. SASLAW

.... eminently readable. It is written throughout in an engaging style with a clear logical development.' The Times HigherEducation Supplement 508 pp. $1987 \quad 0 \quad 521 \quad 349753 \quad$ Pb $\$ 17.50$ net

\section{The Interacting Boson Model}

\section{F. IACHELLO and A. ARIMA}

Describes the mathematical framework on which the interacting boson model of the nucleus is based and discusses the applications of this model to the calculation $\begin{array}{llllllll}\text { of properties of nuclei. } \quad 256 p p . & 1987 & 0 & 521 & 30282 & X & \$ 32.50 & \text { net }\end{array}$

For further information on all books in this series and the whole range of Cambridge Physics titles please write to Sally Seed at the address below.

\section{Cambridge University Press}

The Edinburgh Building, Shaftesbury Road, Cambridge CB2 2RU, England 
Table 2: European millimetre-wave telescopes

\begin{tabular}{|llrr|}
\hline Institute & Location & Diameter & $\lambda$ min \\
IRAM & Pico Veleta (Spain) & $30 \mathrm{~m}$ & $0.8 \mathrm{~mm}$ \\
IRAM & Plateau de Bure (France) & $4 \times 15 \mathrm{~m}$ & $0.8 \mathrm{~mm}$ \\
Chalmers T.U. & Onsala (Sweden) & $20 \mathrm{~m}$ & $2.6 \mathrm{~mm}$ \\
IGN & Yebes (Spain) & $14 \mathrm{~m}$ & $2.6 \mathrm{~mm}$ \\
Univ. Helsinki & Metsaehovi (Finland) & $14 \mathrm{~m}$ & $2.6 \mathrm{~mm}$ \\
\hline
\end{tabular}

The alignment suggests a collimation mechanism which operates over a wide range of dimensions (light years to millions of light years) and time scales (months to tens of millions of years) and channels energy from the nucleus to the outer lobes of the radio source. The fact that the collimation mechanism maintains a constant direction while producing a radio source with energy $10^{54} \mathrm{~J}$ is evidence that gyroscopic action of a massive black hole in the centre may provide the long term stability in orientation.

\section{Geodesy and Geophysics}

Far from being stable, the Earth's crust has seen considerable changes in the geological past and has still not come to rest today, as is evidenced for example by the intense activity in the seismic zones. In fact the pattern of seismic belts has revealed that the crust is fragmented into large crustal blocks kept in relative motion by some as yet poorly understood processes. From paleomagnetic and sea floor spreading data the mean motion of these tectonic plates during the past $\mathbf{2 0 0}$ million years has been inferred to range between one and ten centimetres per year. Geophysists are eager to know more about presentday rates of motion in their effort to improve the understanding of the tectonic processes in the Earth's crust. This new knowledge may eventually contribute to the prediction of earthquakes and volcanic eruptions.

In recent years geodesists have been successful in implementing new measurement techniques such as satellite laser ranging (SLR) and VLBI to achieve accuracies of a few centimetres over continental distances. The most promising results have been obtained with special wide bandwidth VLBI equipment using high delay resolution to determine the vector baselines between radio telescopes in networks around the globe. A series of repeated baseline measurements across the North Atlantic has now revealed a slow separation of the continents of about $1.5 \mathrm{~cm}$ per year, which is in good agreement with the long term continental drift rate derived from geophysical data.

Of course, crustal motions constitute only one of several interesting geodyna- mical phenomena that can be detected with VLBI. Among the most important are:

- polar motion and Earth rotation rate,

- Earth tides.

- global sea level changes,

- vertical uplift and subsidence,

- precession and nutation.

In many of these topics the European continent offers a wide variety of opportunities for fruitful scientific studies. The attention of geophysicists is focussed in particular on southern Europe, where one of the world's most active seismotectonic areas is found. The entire Mediterranean belongs to the highly complex collision zone that lies between the African and Eurasian plates, which explains the frequent occurrence of strong earthquakes.

The VLBI technique with its quasar based reference frame is capable of providing a stable network of fundamental points to support the classical geodetic techniques of triangulation and levelling. The existing radio telescopes in Europe already form a solid basis for such a network (Fig. 5) although there is still a need for stations in the far North and in the Mediterranean.

\section{VLBI in the 1990's}

i) New telescopes/arrays for centimetrewavelength observations

The prospects of getting much more than $30 \%$ of all available time on exis- ting telescopes are rather small because of competition from other programmes, and this has led to proposals, some of them successful, for new telescopes dedicated primarily to VLBI. The prime example of this is the VLB Array in the United States, which has been funded recently and should be completed in 1992 [3]. This is a 10-antenna (each 25 $m$ in diameter) array on United States territory from Hawaii to the Virgin Islands and from near the Mexican border to near the Canadian border. This will be the first dedicated VLBI system in the world providing not only high quality images but also full-time operation, including the possibility for observations of "targets of opportunity" (e.g. unpredictable short timescale flares in stars or active galactic nuclei) which the parttime networks of the present day find hard to accommodate. Joint observations together with the Very-Large-Array VLA $(35 \mathrm{~km}$ baseline interferometer in New Mexico, USA) will ensure excellent $\mathrm{U}-\mathrm{V}$ plane coverage from $100 \mathrm{~m}$ or so to $8000 \mathrm{~km}$.

In Europe, a dedicated VLBI array is a project that must await a greater degree of political cohesion than is presently the case. However there are a number of new large diameter dedicated VLBI telescopes being built by the member institutes (Table 1), which when operating in the EVN of the 1990's will ensure a unique place for European VLBI for extremely high sensitivity VLBI observations. In addition there is the possibility of simultaneous observation with the 200 $\mathrm{km}$ MERLIN array in the UK [4] to provide essentially uniform $U-V$ coverage from $5 \mathrm{~km}$ out to about $2000 \mathrm{~km}$. Additional spacings, out to the maximum possible on Earth, will be generated by coopera-

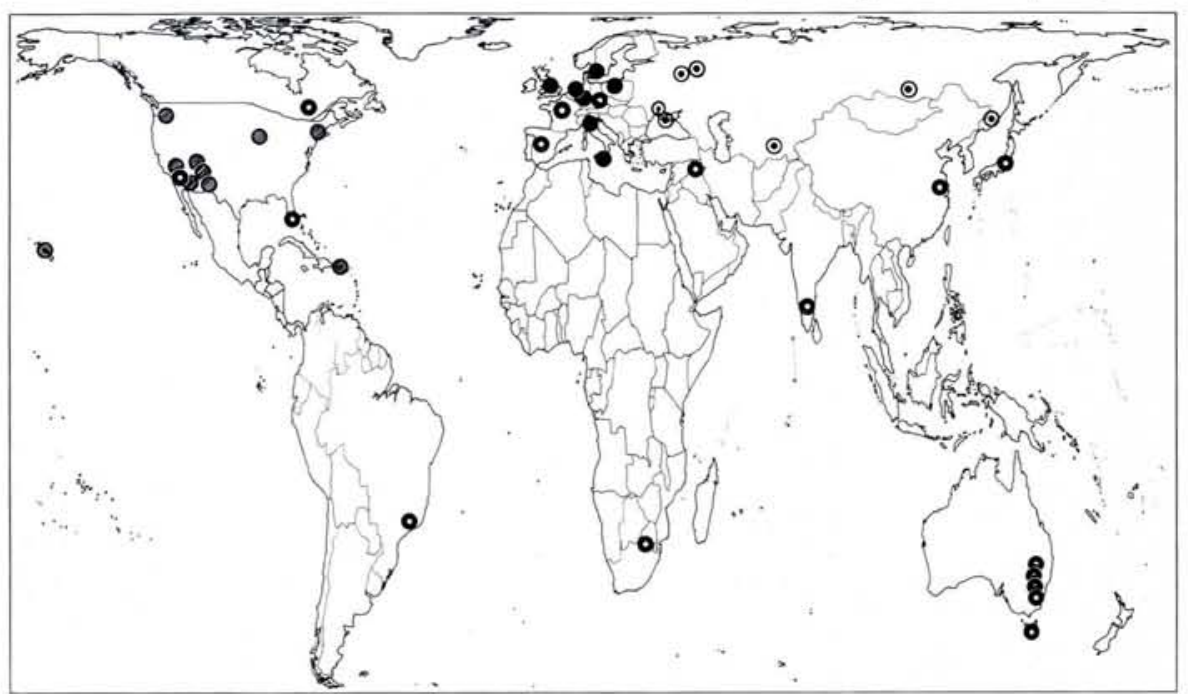

Fig. 5- World map showing radio telescopes expected to be in operation in the early 1990's with substantial observing time available for VLBI. The symbols are as follows: solid circle, European VLBI network; hatched circle, United States dedicated VLB array; open circle with central dot, USSR VLBI network; open circle, other telescopes. 
tive observations with US telescopes as well as with the $70 \mathrm{~m}$ Suffa telescope near Samarkand in Soviet Central Asia. Comparable U-V plane coverage to the VLBA can be achieved, particularly for northern sources.

In China, a 25-m antenna has been constructed near Shanghai and others are planned. In Japan there are plans for geodetic VLBI. In Australia the possibility of extending the Australia Telescope to span the continent is being discussed. Fig. 5 shows all the telescopes expected to be in the early 1990's with substantial observing time available for VLBI.

\section{ii) Millimetre-wavelength VLBI}

Higher angular resolution can be achieved through the use of shorter wavelengths for Earth-based observations (mm-VLBI) and through extension of the baselines by an element or elements in space (see next section). Onsala and MPIfR have participated in local and global observations at $8 \mathrm{~mm}$ wavelength and MPIfR is involved in a proposal to expand European facilities for $\mathrm{mm}$-VLBI at Pico Veleta and Plateau de Bure together with IRAM (Institute for Millimetric Radio Astronomy) in Grenoble.

Table 2 lists the European telescopes capable of participating in a mm-VLBI network.

\section{iii) Space VLBI}

As we have seen, current VLBI networks and the planned VLBA in the USA utilise the whole Earth as a radio telescope to achieve not only sub-milli-arcsecond angular resolution, but also good quality images. The next obvius step for the interferometry technique is to go beyond the confines of the Earth and position an element or elements in Earth orbit.

Apart from the considerable technical challenge of going into space, there are sound scientific reasons for doing so. Foremost amongst these is high quality imaging at increased angular resolution to investigate those sources, and source components, still unresolved with ground-based arrays. In addition, prospects for imaging in the equatorial region of the sky and in the southern hemisphere are significantly enhanced by the presence of a space element which can provide adequate U-V coverage for the first time. "Rapid" imaging on timescales of a few hours for variable galactic sources is also made possible, again because of enhanced U-V coverage compared to ground-based arrays.

Three space VLBI projects are under study at the present time: QUASAT in Europe, VSOP in Japan, and RADIOASTRON in the USSR. RADIOASTRON

\section{Technische Universität Wien}

An der Technisch-Naturwissenschaftlichen Fakultät (Institut für Allgemeine Physik) ist die Planstelle eines

\section{Ordentlichen Universitätsprofessors für Allgemeine Physik (Nachfolge von Prof. F. VIEнвÖск)} zu besetzen.

Voraussetzungen: Habilitation oder gleichzuhaltende wissenschaftliche Eignung in einem Gebiet der Experimentalphysik sowie besondere didaktische Befähigung. Die Vielfalt der am Institut bestehenden Forschungsrichtungen (Oberflächenphysik und -analytik, Plasmaphysik und -technik, Optische Spektroskopie, Physikalische Messtechnik) soll erhalten und gefördert werden. Erfahrung in der Zusammenarbeit mit der Industrie ist erwünscht.

Dienstpflichten: Der/die zu Berufende hat sein/ihr Fachgebiet in Forschung und Lehre angemessen zu vertreten; insbesondere hat er/sie im Rahmen der Grundlagenausbildung für Studierende der Technischen Physik eine fünfstündige dreisemestrige Einführungsvorlesung aus Physik im Zweijahresturnus zu halten sowie die zugehörigen Rechen- und Laborübungen verantwortlich zu leiten.

Bewerbungen sind unter Beifügung eines Lebenslaufes mit eingehender Darstellung des wissenschaftlichen Werdeganges und der bisherigen Lehrtätigkeit sowie vollständiger Verzeichnisse der Publikationen und der gehaltenen Lehrveranstaltungen bis spätestens 31 Mai 1988 an das Dekanat der Technisch-Naturwissenschaftlichen Fakultät der Technischen Universität Wien

(Getreidemarkt 9, A-1060 Wien, Austria) zu richten.

\section{The Foundation for Fundamental Research on Matter}

promotes fundamental scientific research on matter in the Netherlands generally and at universities in particular. The Foundation tries to achieve this purpose by stimulating research in new fields of physics, by co-ordinating existing research projects and by involving its institutes and working groups in the education and training of young physicists.

\section{The Foundation has an opening for an}

\section{Experimentalist in Intermediate-Energy Nuclear Physics} at the University of Utrecht (the Netherlands).

The group invites applicants for a tenure-track position in the field of intermediate-energy nuclear physics with electromagnetic probes. At present the group is involved in both nuclear-structure physics mainly with e.m. probes and heavy-ion physics. The group consists of about 30 physicists (experimentalists and theoreticians) and 20 technicians.

The successful candidate is expected to participate in the ongoing programme and to develop novel lines of research in particular at the $500 \mathrm{MeV}$ electron accelerator of the nearby National Institute for Nuclear and High-Energy Physics (NIKHEF) in Amsterdam. An important extension of this facility towards $100 \%$ duty-factor electron beams with a maximum energy of $800 \mathrm{MeV}$ will be commissioned within the next four years. A key project of the candidate would be to develop and implement a multi-element state-of-the-art neutron detector system to be used at the NIKHEF facility and at other facilities abroad. He is expected to lead a team of graduate students and postdocs. He should preferably have experience with research involving accelerators and large scale detection apparatus. In addition, participation in teaching undergraduate and graduate students is expected.

Applicants should have a Ph.D., preferably in experimental intermediate-energy nuclear physics with a period of postdoctoral experience.

For further information please contact:

Prof. Dr. P.K.A. de Witt Huberts, Tel.: (20) 5922145 / 5922143 or Prof. Dr. C. van der Leun, Tel. (30) 532512 / 531492.

Applications should reach the Personnel Division of the Physics Faculty of the University of Utrecht,

Postbus 80.000, NL-3508 TA Utrecht, the Netherlands. 
is an approved programme with a launch expected in 1992-3, QUASAT and VSOP are at different stages in the pre-selection process but could be launched round 1995.

RADIOASTRON involves a $10 \mathrm{~m}$ diameter antenna in a 24-hour orbit around the Earth, achieving baseline lengths up to about $80000 \mathrm{~km}$. At the highest frequency of observation $(22 \mathrm{GHz})$, the angular resolution approaches 25 microarcsec. With such an orbit, high quality will not be possible due to gaps in the U-V plane coverage, so the scientific thrust will be towards detecting, rather than imaging, compact structures in a variety of sources. This aim is complementary to that of QUASAT which envisages providing high quality imaging at somewhat less angular resolution ( $\cong 60$ micro-arcsec at $22 \mathrm{GHz}$ ).

The European Consortium for VLBI is jointly financing the construction of two space-qualified, coolable, $5 \mathrm{GHz}$ receivers for RADIOASTRON. The NFRA in Dwingeloo is managing the overall receiver project and building the mixer/IF units; the MPIfR is building the low noise amplifiers. ESA/ESTEC is providing advice during the course of the project, as well as the use of environmental test facilities for testing prototypes.

QUASAT was proposed to ESA as a joint ESA-NASA space VLBI mission in November 1982 by an international group of astronomers. An ESA Assessment Study was carried out from October 1983 to June 1985 in parallel with a pre-Phase A study in NASA. QUASAT was approved for Phase A (industrial feasibility) study in Europe in February 1986.

The aftermath of the Challenger crash in January 1986 forced NASA to delay consideration of new projects in the Explorer program, including QUASAT, and caused ESA to re-evaluate the cooperative framework under which QUASAT had been studied up to that time. This reevaluation resulted in a mission concept with ESA as the leading agency but with substantial support from JPL, and Australian and Canadian space interests. This new concept was approved for an ESA Phase A in February 1987, and is now being carried out by an industrial consortium headed by Aeritalia in Turin.

In the meantime in 1986, a successful demonstration of space VLBI was made at the first attempt using the NASA TDRSS satellite in geostationary orbit together with $64 \mathrm{~m}$ antennas in Japan and Australia [5]. This result emphasised that space VLBI is a viable, and potentially very valuable, space astronomy discipline.
QUASAT is to be a free-flying satellite carrying a $15 \mathrm{~m}$ class radio telescope in elliptical orbit around the Earth. The radio telescope will be deployed in space, and used to make interferometric observations of radio sources in conjunction with the major ground-based VLBI arrays in W. Europe, USA, Australia and the USSR. The mission design lifetime is two years, but an operational lifetime of five years is expected.

The space-borne antenna will be capable of observing in both hands of circular polarization simultaneously at any two of the frequency complement of $22,5,1.6$ and $0.3 \mathrm{GHz}$, and will relay the received signals via a digital or analogue link directly to telemetry stations (NASA DSN and/or ESA) on the ground. The bandwidth of the dual polarization link will be at least $32 \mathrm{MHz}$ in each hand of polarization. A phase/frequency reference for the antenna in space, stable to about $1 \times 10^{-14}$, will be based on hydrogen maser oscillators on the ground and relayed directly to the satellite via a two-way link from the telemetry stations in turn. All communication with the space element will be through one or more telemetry stations in the network.

After transmission to the ground, the signal will be recorded on magnetic tape in digital form, and transported to the central processing facility of the European or US VLBI array for correlation with similar tapes from the ground VLBI arrays. After correlation and calibration the data will be sent to the principal investigators for further analysis.

Two antenna configurations have been studied; in ESA a 15 meter diameter inflatable space rigidized structure developed by Contraves, Switzerland, and in NASA a 15 meter wrap-rib deployable mesh structure developed by the Lockheed Corporation, California. Both appear to be satisfactory for the mission. Other areas of development are the radio astronomy feeds and an active cooling system for the radio astronomy receivers. An industrial study of the feeds has shown good performance for a compact three frequency co-axial feed at prime focus which can also accommodate a dipole array for $327 \mathrm{MHz}$. The radio astronomy receivers will be based on HEMT amplifiers, cooled actively by Stirling cycle coolers.

\section{iv) European Data Processing Facility for 1990s}

To meet the obvius scientific challenges of the 1990s, the EVN will need to provide better image quality, higher spectral resolution, higher frequency capability, and be able to handle data from a space element. The European
Consortium is seeking funding for an augmentation of the EVN facilities in the 1990 s through the European Communities' "Framework Programme of Technological Research and Development (1987-1991)".

The proposal has two major components: the Data Processing Facility (DPF) including the Data Playback System (DPS), and the Data Acquisition System (DAS). The current concept for the processing facility centres around a correlator capable of processing up to $256 \mathrm{Mbits} / \mathrm{s}$ data in one polarisation from 16-stations simultaneously. The correlator is of the "X-F" type and will employ state-of-the-art $2 \mu \mathrm{CMOS}$ technology.

The DAS and DPS will follow the US VLBA design for obvious compatibility reasons. The 24 acquisition terminals and 18 playback terminals envisaged are to be built in Europe.

The NFRA in Dwingeloo will carry the overall project responsibility and will construct the DPF. The MPIfR in Bonn will manage the construction of the DAS and DPS.

\section{Acknowledgement}

I wish to thank Prof. J. Campbell (University of Bonn) for contributing the section on geodesy and geophysics.

\section{REFERENCES}

1. Biretta J.A. and Cohen M.H., "Investigations of $3 \mathrm{C} 345^{\prime \prime}$ in Superluminal Radio Sources, Eds. J.A. Zensus, T.J. Pearson (CUP, Cambridge) p. 40.

2. Schilizzi R.T., "Observational Evidence for Outflow in Active Galactic Nuclei and in Xray Binaries" in "Mass Outflows in Stars and Galaxies", Eds. R. Gilmozzi and L. Bianchi (Reidel, Dordrecht) in press.

3. Kellermann K.I. and Thompson A.R., "The Very Long Baseline Array", Science 229 (1985) 123.

4. Davies J.G., Anderson B. and Morrison I., "The Jodrell Bank Radio-Linked Interferometer Network" Nature 288 (1980) 64.

5. Levy G.S. et al., "Very Long Baseline Interferometric Observations Made with an Orbiting Radio Telescope" Science 234 (1986) 187.

\section{Basic interferometry theory}

Thompson A.R., Moran J.M. and Swenson G.W., Interferometry and Synthesis in Radio Astronomy (Wiley and Sons) 1986, 534 pp.

\section{General VLBI}

Carter W.E. and Robertson D.S., "Studying the Earth by Very Long Baseline Interferometry" Sci. Amer. 255 (1986) 44.

Readhead A.C.S., "Radio Astronomy by Very Long Baseline Interferometry" Sci. Amer. 246 (1982) 38.

Schilizzi R.T., "Very Long Baseline Interferometry" Radio Science 21 (1986) 665. 